\title{
UTILIZACIÓN DE COLIGÜE (Chusquea culeou) EN LA FABRICACIÓN DE PULPA QUÍMICA
}

\section{SANDRA K. RODRÍGUEZ (*) y MARCO TORRES (**)}

(*) Ingeniero Civil Químico, MSc. Celulosa y Papel.

(**) Técnico en Celulosa y Papel

Intituto de Tecnologia de Productos Forestales, Universidad Austral de Chile, Casilla 567, Valdivia.

\section{RESUMEN}

Para este trabajo se procesaron muestras de coligue (Chusquea culeon Desv.) que constituye un material abundante en los bosques de la zona Sur de Chile, para la obtención de pulpa Kraft cruda.

El estudio consideró variación de la carga de álcali efectivo (14 y 18\%), de la temperatura máxima $\left(160\right.$ y $\left.170^{\circ} \mathrm{C}\right)$ y del tiempo a temperatura máxima (15 y 30 minutos).

Los resultados obtenidos demuestran que el rendimiento del proceso varia entre 46 y $55 \%$. para número kappa entre 7 y 20. Las propiedades de resistencia resultan muy similares entre las pulpas obtenidas.

Para las condiciones ensayadas las pulpas resultantes presentan propiedades fisicomecánicas inferiores a aquellas que se obtienen a partir de madera de coniferas y algunas latifoliadas.

Palabras Clave: Pulpa Quimica, Chusquea culeou

\section{ABSTRACT}

Samples of Chusquea culeou Desv: were processed to obtain unbleached kraft pulp since they constitute an abundant material in the south of Chile.

The study considered the variation in alkali (14 and 18\%), the maximum temperature of pulping $\left(160\right.$ and $\left.170^{\circ} \mathrm{C}\right)$ and the time at maximum temperature (15 and 30 minutes). The results show that the total yield of the process ranges between 46 and 55\% and the Kappa number between 7 and 20. The strenght properties of the two pulps produced were similar.

At the conditions experimented the pulps obtained show physical and mechanical properties lower than those obtained from softwoods and several hardwoods.

Keywords: Chenical Pulp. Chusquea culeon 


\section{INTRODUCCIÓN}

La industria mundial de la pulpa y el papel ha adquirido clara conciencia de la necesidad de ampliar sus fuentes de recursos fibrosos. No hay duda respecto a que las fibras no maderables jugarán un papel importante en la industria, las ya existentes crecerán y se desarrollaran otras fibras no tradicionales, especialmente en los países del tercer mundo, los que deberán incrementar la producción de pulpa con el fin de suplir las necesidades de sus mercados internos.

Las estadisticas de la FAO muestran que el crecimiento de la producción de la producción de pulpa a partir de fibras no maderables para el período 1976 a 1981 fue de $3,8 \%$ en contraste con el $1,9 \%$ para la pulpa a partir de fibras maderables.

Por otra parte la producción mundial de pulpa, papel y cartón usando fibras no maderables, se ha incrementado de 5,4 millones de toneladas en 1972 a cerca de 12 millones de toneladas en 1990, en otras palabras, se ha duplicado (Dorado, 1988; Jeyasingham, 1991 ).

En Bangladesh el bambú es una materia prima importante para uso rural e industrial. Cubre una superficie total de 600.000 ha con un rendimiento anual de aproximadamente 1 millón de toneladas. Se han realizado estudios tendientes a determinar las caracteristicas fisicas y quimicas de distintas especies y sus aptitudes pulpables, determinándose que las mejores calidades de pulpa se obtienen desde Bambusa tulda, Melocanna baccifera, Oxytenanthera nigrociliata, Neohouseana dulloa de 6 meses de edad y Dendrocalamus lognispathus de 12 meses. Se obtuvieron rendimientos de pulpa entre 43 y $52 \%$ con indices Kappa entre 15 y 24 . Los valores máximos de ruptura, explosión y rasgado fueron de $10 \mathrm{~km}, 91$ y 215 , respectivamente, a 250 CSF (Ali, 1981).

Numerosas publicaciones (Guha y Pant, 1966; Bhola, 1976; Negi, 1970; Guha et al., 1975 ) indican que los bambúes podrian ser pulpados usando $14-15 \%$ de álcali total y $25 \%$ de sulfidez para producir pulpas ( $40 \%$ rendimiento) adecuadas para la fabricación de papeles de impresión y escritura.

Estudios de Singh et al. (1976) sobre morfologia de la fibra y resistencia de la pulpa de doce especies de bambúes. revelaron que las fibras son altamente heterogéneas en relación al ancho de lumen. longitud y ancho de fibras y proporciones de parénquima las cuales resultaron en diferencias en las propiedades fisicas de las pulpas sin batir. Los autores no encontrararon una relación entre características de las fibras y propiedades de resistencia del papel. Dado que existen grandes variaciones dentro de las especies, las características de la fibra no pueden ser usadas como un criterio para la clasificación de los bambúes para la producción de papel. 
En Chile existe una gran producción de biomasa aérea de Chusquea culeou (Veblen, Schelegel y Escobar, 1974) lo cual hace interesante estudiar su posible utilización industrial.

Rijo et al (1987) realizaron un estudio de algunas características básicas necesarias para determinar la aptitud de esta especie como materia prima industrial. Los autores determinaron una densidad promedio de Chusquea culeou de $0,584 \mathrm{~g} / \mathrm{cm}^{3}$, la que se encuentra dentro de los rangos de las especies normalmente usadas en las industrias de la madera. En cuanto al largo de fibras, ésta registró valores promedios entre 1,4 y 2,2 $\mathrm{mm}$ los que se encuentran cercanos a los máximos que presentan las especies latifoliadas. En la composición química se encontró que la proporción con que participan los compuestos principales es semejante a la madera de latifoliadas, observándose una cantidad de lignina relativamente baja (20\%). El contenido de cenizas es notablemente superior $(1,5 \%)$ a los valores en las maderas de diferentes especies.

Se desarrolló el presente trabajo, para estudiar la aptitud del coligue como materia prima para la producción de pulpa kraft.

\section{MATERIAL Y MÉTODO}

Para la realización del estudio se usó como material de ensayo. muestras de edad superior a un año, con lo cual se garantizó que hubiese finalizado su proceso de lignificación. El material fue el mismo que utilizaron Rijo et al. (1989) en la determinación de las características básicas de la especie.

Las muestras de coligue fueron transformados en astillas en un astillador industrial facilitado por la industria INFODEMA en Valdivia. Las astillas producidas fueron tamizadas según norma TAPPI T 16 ts-61, en un harnero Williams de perforaciones circulares, y se seleccionó para pulpaje la fracción entre $3 / 16$ y $7 / 8$ de pulgada.

En la realización de los pulpajes kraft se trabajó con un digestor de laboratorio $\mathrm{M} / \mathrm{K}$ System de $6.5 \mathrm{~L}$.

En las cocciones se usaron $700 \mathrm{~g}$ de astillas con una humedad de $14,6 \% \mathrm{bh}$. La relación licor/madera y la sulfidez se mantuvieron constantes, siendo éstas de $4 / 1$ y $25 \%$ bms, respectivamente.

Se variaron la carga de álcali efectivo. la temperatura máxima y los tiempos hasta y a temperatura máxima. Los valores asignados a estas variables se muestran en el Cuadro $\mathrm{N}^{\circ} 1$.

Como respuestas del pulpaje se midieron: Rendimiento clasificado, rechazos y Kappa, para la pulpa; y álcali residual y porcentaje de sólidos. para el licor negro. También se realizó la clasificación Clark de fibras. 
Cuadro $N^{\circ} 1$.

CONDICIONES DE PULPAJE

\begin{tabular}{|c|c|c|c|c|c|}
\hline Pulpaje & Alcali efectivo & Temperatura & \multicolumn{2}{|c|}{ Tiempo (min) } & Factor \\
\cline { 4 - 5 } $\mathrm{N}^{\circ}$ & $(\%$ obms) $(\mathbf{o x})$. & $\left({ }^{\circ} \mathrm{C}\right)$ & (hasta $\left.\mathrm{T}^{\circ}\right)$ & $(\mathrm{a} \mathrm{T})$ & " \\
\hline 1 & 18 & 170 & 100 & 15 & 431 \\
2 & 18 & 170 & 100 & 30 & 661 \\
3 & 14 & 170 & 100 & 15 & 431 \\
4 & 14 & 170 & 100 & 30 & 661 \\
5 & 14 & 160 & 100 & 15 & 195 \\
6 & 14 & 160 & 100 & 30 & 294 \\
7 & 14 & 160 & 90 & 2 & 101 \\
\hline
\end{tabular}

Se batieron las pulpas obtenidas en los pulpajes $\mathrm{N}^{\mathrm{3}} 3$ y 5 correspondientes a las temperaturas máximas de cocción de 170 y $160^{\circ} \mathrm{C}$, respectivamente (Cuadro $\mathrm{N}^{\circ} 1$ ).

Las pulpas se refinaron en una batidora Valley según Tappi T 200 om-85. A continuación se midieron las siguientes propiedades en hojas de ensayo de $60 \mathrm{~g} / \mathrm{m}^{2}$, fabricadas con pulpas a diferentes grados de refinación: Densidad. Longitud de Ruptura. Factor de Rasgado y Factor de Explosion.

\section{RESULTADOS Y DISCUSIÓN}

\section{Respuestas del Pulpaje}

Los resultados del pulpaje se muestran en el Cuadro $\mathrm{N}^{\circ} 2$.

\section{Cuadro $\mathrm{N}^{\circ} 2$.}

RESPUESTAS AL PROCESO DE PUI,PAJE

\begin{tabular}{|c|c|c|c|c|c|c|c|c|}
\hline \multirow[b]{2}{*}{$\begin{array}{c}\text { Pulpaje } \\
\mathrm{N}^{\circ}\end{array}$} & \multirow[b]{2}{*}{$\begin{array}{c}\text { Factor } \\
\text { "H" }\end{array}$} & \multirow{2}{*}{$\begin{array}{c}\text { Rendim.. } \\
\text { Clasif } \\
(\% \mathrm{bms})\end{array}$} & \multirow[b]{2}{*}{$\begin{array}{l}\text { Rechazo } \\
\text { (\%obms) }\end{array}$} & \multirow{2}{*}{$\begin{array}{c}\text { Rendim. } \\
\text { Total } \\
\text { (\%bms) }\end{array}$} & \multirow[b]{2}{*}{$\begin{array}{l}\text { Índice } \\
\text { Kappa }\end{array}$} & \multicolumn{2}{|c|}{ Alcali } & \multirow{2}{*}{$\begin{array}{l}\text { Sólidos } \\
\text { Totales } \\
(\% \mathrm{p} / \mathrm{v})\end{array}$} \\
\hline & & & & & & $\begin{array}{c}\text { Residual } \\
(\mathrm{g} / \mathrm{L})\end{array}$ & $\begin{array}{c}\text { Consumido } \\
\text { (\%bms) }\end{array}$ & \\
\hline 1 & 431 & 44,8 & 1.4 & 46,2 & 8.8 & 14.3 & 21,6 & 21,6 \\
\hline 2 & 661 & 46.2 & 0,5 & 46.7 & 7,4 & 13,4 & 21,7 & 21,7 \\
\hline 3 & 431 & 46,3 & 4.3 & 50.6 & 13,2 & 6.7 & 19.1 & 19,1 \\
\hline 4 & 661 & 48,5 & 2.5 & 51.0 & 10,6 & 6,1 & 18,7 & 18,7 \\
\hline 5 & 191 & 47.2 & 8.0 & 55.2 & 19.6 & 10,4 & 18,7 & 18,7 \\
\hline 6 & 294 & 50.3 & 3.2 & 53,5 & 14,1 & 7,7 & 19,2 & 19,2 \\
\hline 7 & 101 & 48.6 & 5.8 & 54.4 & 20,4 & 11.2 & 19,1 & 19,1 \\
\hline
\end{tabular}

Se alcanzaron rendimientos clasificados entre 44 y $50 \%$ e indices Kappa entre 7 y 20 (Cuadro $\mathrm{N}^{\circ} 2$ ).

El efecto de disminuir la concentración de álcali efectivo de 18 a $14 \%$ significó un aumento en todas las respuestas del pulpaje. es decir rendimiento clasificado, rechazos 
y Kappa. El porcentaje de sólidos totales disminuyó debido a la menor disolución de lignina.

Al aumentar el factor $\mathrm{H}$ desde 431 a 661 se observó un aumento en el rendimiento clasificado de entre 1,4 a 2,2 \%, dependiendo de la concentración de álcali.

En general con todas las condiciones de cocción ensayadas se obtuvieron valores aceptables de rendimiento y alto grado de delignificación de las pulpas, lo cual era de esperar por el bajo contenido de lignina de la materia prima.

\section{Clasificación Clark de Fibras}

En el Cuadro $\mathrm{N}^{\mathrm{o}} 3$ se muestra la Clasificación Clark de fibras.

\section{Cuadro $\mathrm{N}^{\circ} 3$}

CLASIFICACIÓN CLARK DE FIBRAS

\begin{tabular}{|c|c|c|}
\hline $\begin{array}{c}\text { Mallas } \\
\text { (Mesch) }\end{array}$ & $\begin{array}{c}\text { Pulpa } \mathrm{N}^{\circ} 3 \\
\text { Factor } \mathrm{H} 431 \\
\text { (\% peso) }\end{array}$ & $\begin{array}{c}\text { Pulpa } \mathrm{N}^{\circ} 5 \\
\text { Factor } \mathrm{H} 195 \\
\text { (\% peso) }\end{array}$ \\
\hline 14 & 1,4 & 0.8 \\
30 & 41,6 & 36,9 \\
50 & 20,8 & 22,3 \\
100 & 9,8 & 1,3 \\
$>100$ & 26,6 & 38,7 \\
\hline
\end{tabular}

A menor factor $\mathrm{H}$ se obtuvo una pulpa con mayor porcentaje de finos, dado por la fracción $\mathrm{M}>100$.

\section{Propiedades Físico-Mecánicas de las Pulpas}

Tiempo de Batido

En la Figura $N^{\circ} 1$ se muestra el tiempo de batido de las pulpas versus drenaje. Con menor factor $\mathrm{H}$ se requirió mayor tiempo de batido, lo cual pudo deberse al mayor contenido de lignina residual presente en esta pulpa. Los tiempos de batido son inferiores a los requeridos para pulpas kraft de coniferas y latifoliadas para alcanzar un grado de batido de $30^{\circ} \mathrm{SR}$. 


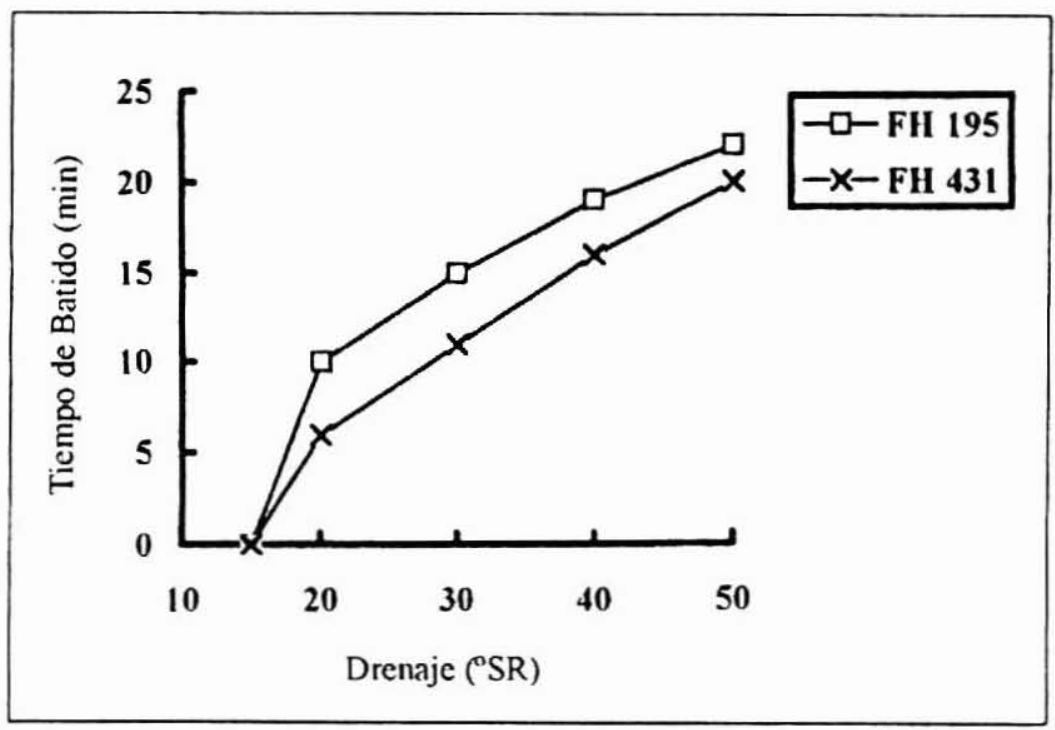

Figura $N^{\circ} 1$. TIEMPO DE BATIDO VERSUS DRENAJE PARA PULPAS CON DISTINTO FACTOR H

Densidad de Hojas

En la Figura №2 se muestra la densidad de hojas versus drenaje. La densidad se relaciona con el grado de enlace entre las fibras. La pulpa de factor H 195 tuvo mayor densidad de hojas debido a la mayor cantidad de finos (fracción de fibras $M>100$ ) y menor porcentaje de fibras largas retenidas en las mallas 14 y 30 . dada por la clasificación Clark (Cuadro No3).

\section{Factor de explosión}

El factor de explosión se muestra en la Figura $\mathrm{N}^{0} 3$. No se observaron diferencias significativas de esta propiedad entre ambas pulpas obtenidas con diferente factor $\mathrm{H}$, mostrando un comportamiento normal. 


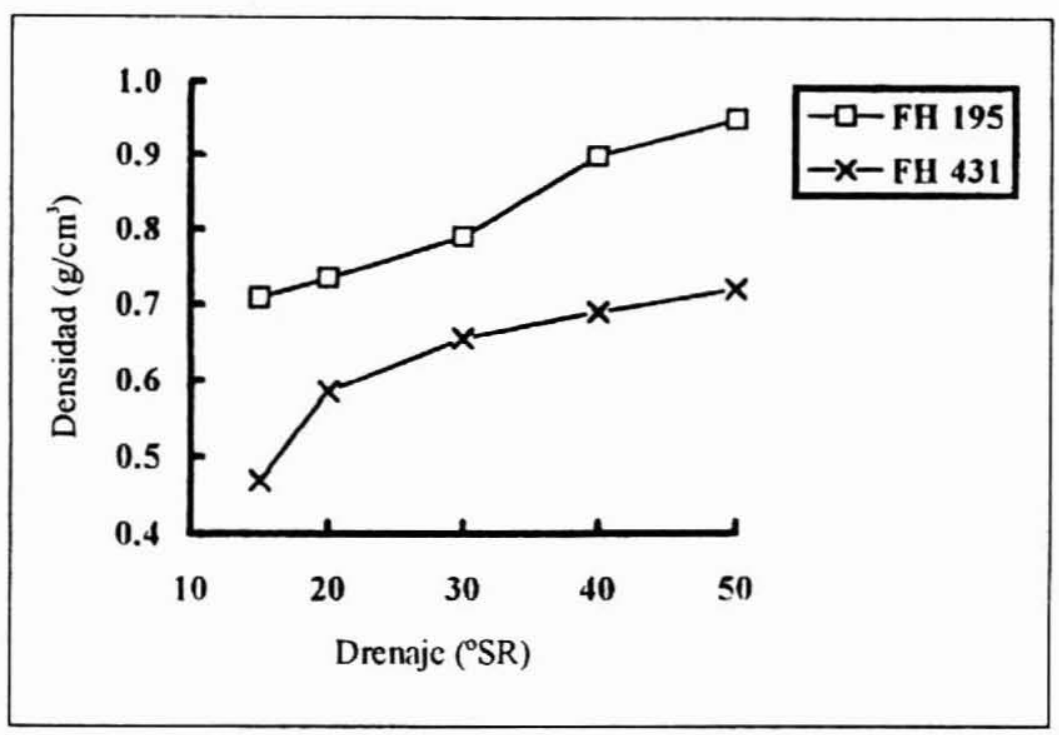

Figura No2. DENSIDAD DE HOJAS VERSUS DRENAJE PARA PULPAS CON DISTINTO FACTOR H

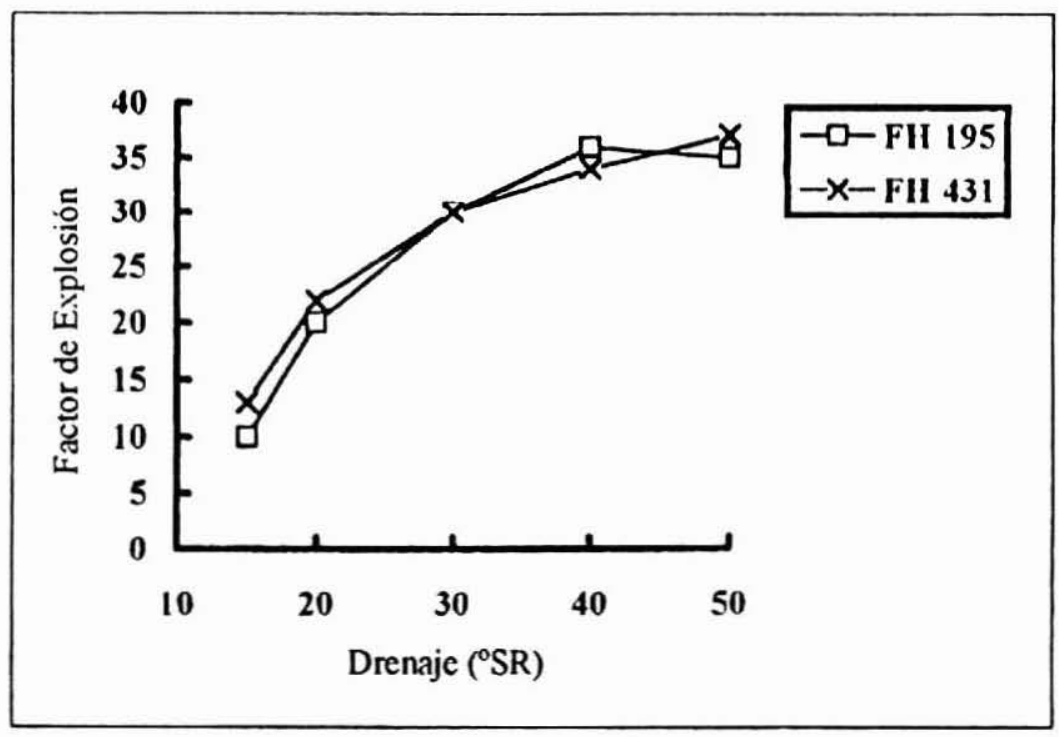

Figura No3. FACTOR DE EXPLOSIÓN VERSUS DRENAJE PARA PULPAS CON DISTINTO FACTOR H 
El valor máximo de factor de explosión fue de 37 a $50^{\circ} \mathrm{SR}$, el cual es inferior a los obtenidos con pino insigne y algunas latifoliadas (Paz et al, 1987).

Longitud de Ruptura

La longitud de ruptura versus el drenaje se muestra en la Figura N 4 . La pulpa con factor $\mathrm{H} 431$ desarrolla con el batido una longitud de ruptura superior en un $15 \%$, a Schopper mayor que 30. comparada con la pulpa de factor H 195 . Esto se puede atribuir al mayor enlace entre fibras obtenido en la primera.

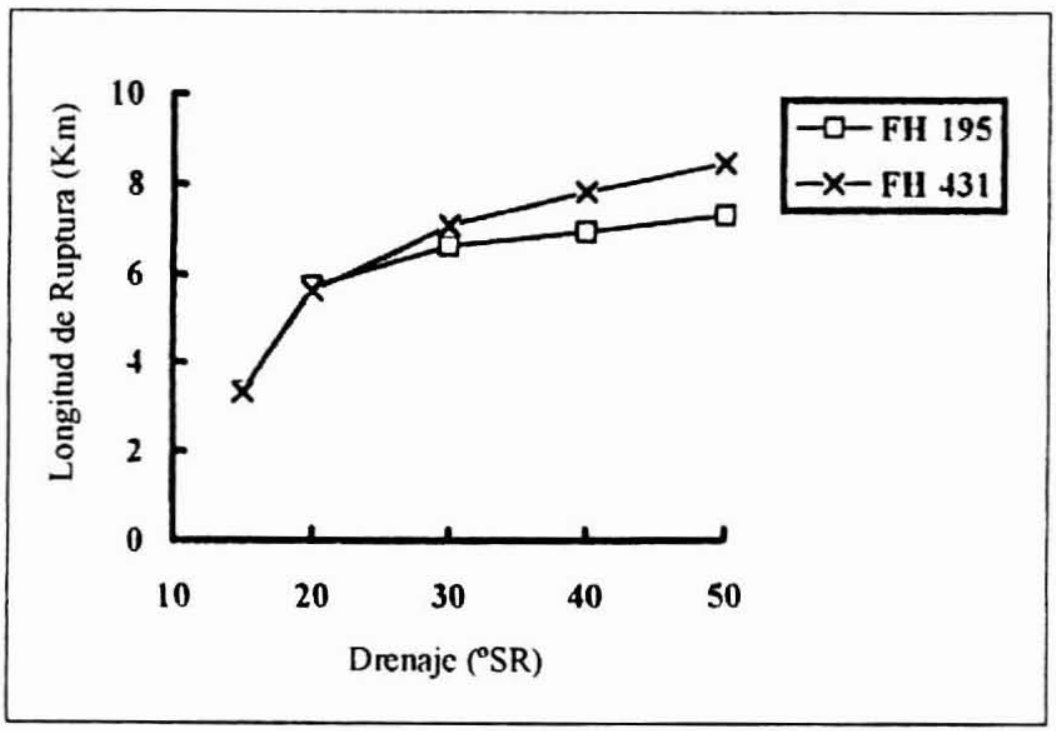

Figura N ${ }^{\circ}$. LONGITUD DE RUPTURA VERSUS DRENAJE PARA PULPAS CON DISTINTO FACTOR H

Los valores máximos de longitud de ruptura se alcanzaron a $50^{\circ} \mathrm{SR}$ y fueron de 8.5 y $7.3 \mathrm{~km}$ para las pulpas $\mathrm{N}^{\circ} 3$ y 5 , respectivamente.

\section{Factor de Rasgado}

El factor de rasgado versus el drenaje se muestra en la Figura No5. A mayor factor $\mathrm{H}$ se obtuvo mayor rasgado, lo cual concuerda con el mayor porcentaje de fibras largas presentes en esta pulpa (Cuadro $\mathrm{N}^{\mathrm{3}} 3$ ). Con ambas pulpas se obtuvo un factor de rasgado máximo a $30^{\circ} \mathrm{SR}$. después del cual éste decrece. 


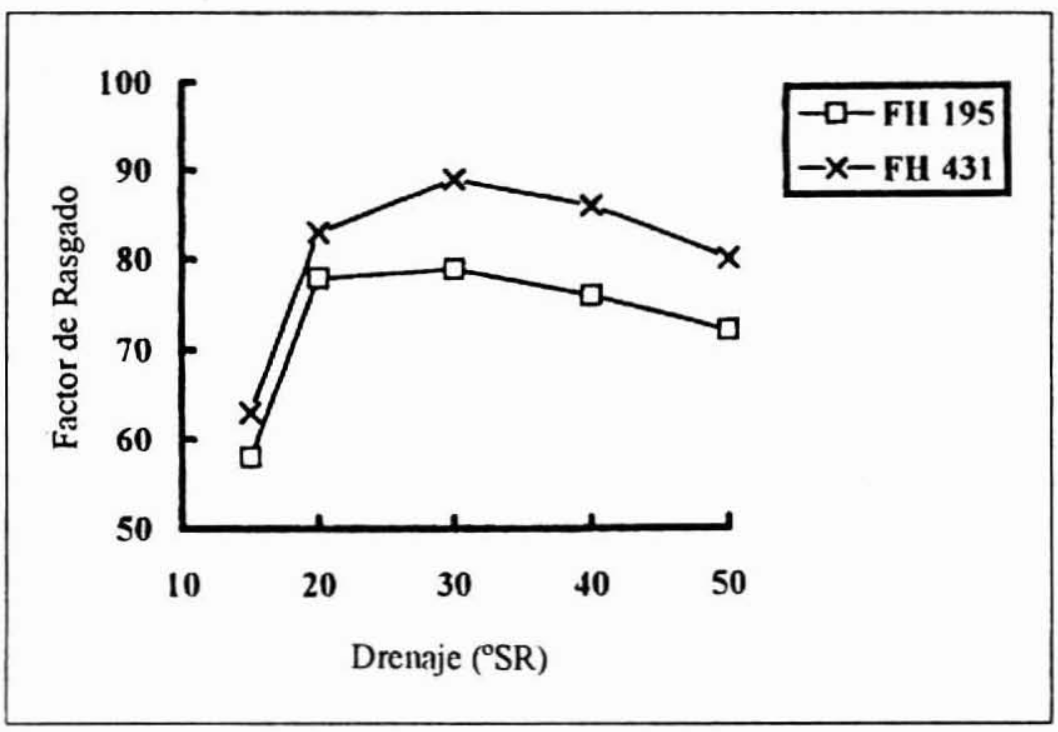

Figura $N^{\circ} 5$. FACTOR DE RASGADO VERSUS DRENAJE PARA PULPAS CON DISTINTO FACTOR H

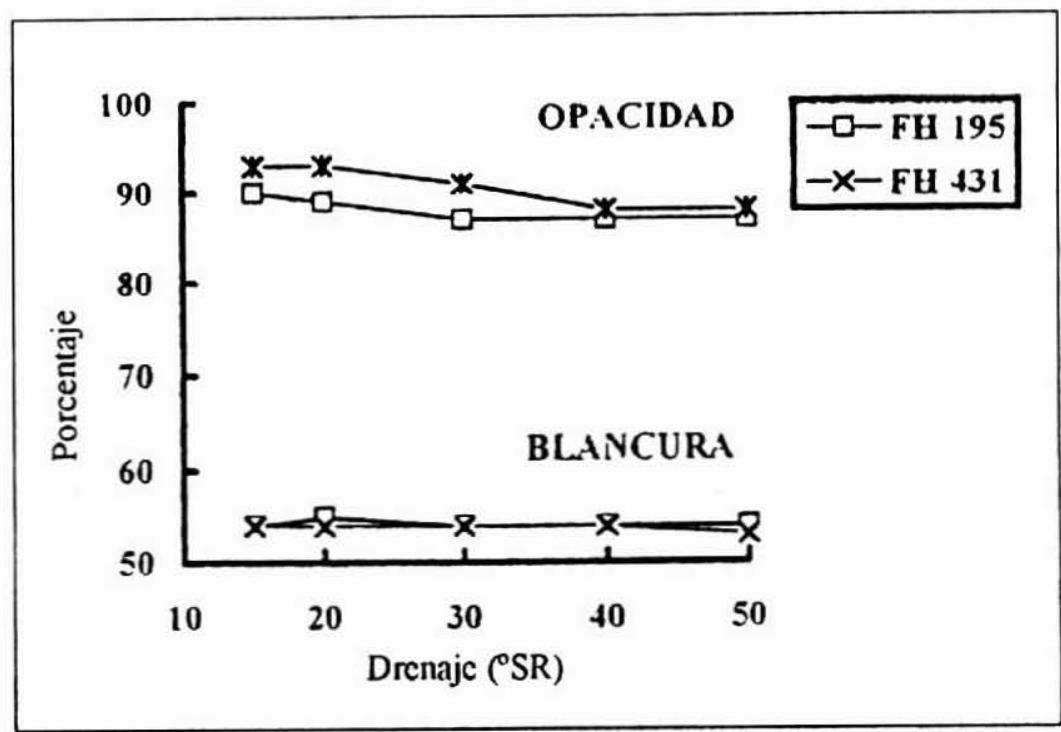

Figura N ${ }^{\circ} 6$. OPACIDAD Y BLANCURA VERSUS DRENAJE PARA PULPAS CON DISTINTO FACTOR H 
La Figura N ${ }^{\circ} 6$ muestra la blancura y opacidad de las pulpas versus drenaje. La blancura fue de $54 \%$ (Elrepho) para ambas pulpas; este valor es superior al que se alcanza con madera de coniferas y latifoliadas (Urzua et al, 1982). La opacidad varió entre 88 y $93 \%$, siendo levemente superior para la pulpa de factor H 431 . Para ambas pulpas la opacidad tiende a dismunir con el batido como es usual para esta propiedad.

\section{CONCLUSIONES}

El coligüe presenta características físicas y quimicas aptas para la producción de pulpa kraft. Los rendimientos de pulpaje son similares a los obtenidos con madera, con indices Kappa inferiores. El alto contenido de células parenquimáticas podria presentar una desventaja en la fabricación de papeles debido a que imparten una menor velocidad de drenaje a la pulpa. El bajo contenido de lignina que presenta la especie representa una ventaja frente a la madera pues requiere menor tiempo de cocción y menor carga de reactivos.

Las propiedades de resistencia de las pulpas en general son inferiores a aquellas que se obtienen a partir de madera mediante un proceso químico. Sin embargo son similares a las propiedades de una pulpa mecánica obtenida a partir de coniferas. No se descarta la posibilidad que esta especie pueda ser utilizada en mezcla con pulpas kraft obtenidas a partir de madera en la formulación de cierta clase de papeles. Por otra parte dado el bajo contenido de lignina del material, también seria interesante un estudio de la factibilidad de producir pulpa semiquimica a partir de éste.

\section{REFERENCIAS BIBLIOGRÁFICAS}

Ali, M. O., 1981. Research and Development on the Production and Utilization of Bamboo in Bangladesh. Proceedings of the Congress Group 5.3 A. Production and Utilization of Bamboo and Related Species, XII IUFRO World Congress Kyoto, Japan, September, pp 6-17.

Bhola, P. P., 1976. Pulping Studies of Hill Jatti Bamboo (B. tulda) from Chachav Hills. Indian Forester, 102(4):242-246.

Dorado, O., 1988. La Fibra de Caña de Azicar como Materia Prima para Fabricación de Papel y Cartón. Acotepac, 2(3):32-39

Guha, S. R. D. and Pant, P. C., 1966. Sulphate Pulping of Phyllostachys bambusoides. Indian Forester, 92(7):467.

Jeyasingham, J., 1991. Experience with the Use of Agricultural Residues for Papermaking. World Pulp and Paper Technology 1991. Sterling Publications International Limited, 49-51.

Negi, J. S., 1970. Effect of Hemicelluloses on Papermaking Properties of Bamboo (D. giganteus). Indian Forester, 101(5):296-300.

Rijo, C., Poblete, H. Diaz-Vaz, J. E., Torres, M. Fernández, A., 1987. Estudio de Algunas Caracteristicas Anatomicas, Fisicas y Quimicas de Chusquea culeou (coligue). Bosque, 8(1):59-61. 
Urzua, D., Aguilar, J., Diaz-Vaz, e., Guerrera, G., Pereda, C., Vergara, C., Inzunza, L, Torres, M, 1982. Utilización Silvoagropecuaria de Terrenos de Ñadi. Aspectos Tecnólogicos de las Maderas de Ñadi. Informe de Convenio N 54,156 p. Universidad Austral de Chile, Facultad de Ciencias Forestales, Serie Técnica, Valdivia, Chile.

Veblen, T., Schlegel, F., Escobar, B. 1979. Biomasa y Producción Primaria de Cusquea culeou Desv. y Chusquea tenuiflora Phil. en el Sur de Chile. Bosque, 3(1): 47-56. 